\title{
Long-term clearance from small airways in patients with cystic fibrosis
}

\author{
M. Lindström**\#, P. Camner`, R. Falk, L. Hjelte", K. Philipson` and M. Svartengren*
}

ABSTRACT: Impaired mucociliary clearance is a hallmark of cystic fibrosis (CF). Early morphological changes first appear in the small airways. Lung clearance was investigated in 11 young CF adults with mild-to-moderate lung disease using a method depositing particles mainly in the small airways.

Radiolabelled Teflon particles $(6 \mu \mathrm{m})$ were inhaled with an extremely slow inhalation flow, $0.05 \mathrm{~L} \cdot \mathrm{s}^{-1}$. Lung retention was measured immediately following inhalations and, on four occasions up to 21 days. The results were compared with data from healthy subjects.

The lung retention at $24 \mathrm{~h}$ in \% of deposition was $67 \%$ (95\% confidence interval $58-76)$ in the CF patients, compared to $48 \%(42-53)$ in the healthy subjects. Clearance on days 1-7 was larger in the CF patients, $22 \%$ (15-29) compared to the healthy subjects, $14 \%(12-16)$. No difference was observed between the CF patients and the healthy subjects in the slow clearance phase at day 7 to day 21, representing small airway clearance. Impaired mucociliary clearance in CF patients results in increased $24-h$ retention and a prolonged rapid clearance phase.

The results of the study do not support the current authors' hypothesis that clearance from small airways is slower in cystic fibrosis patients compared to healthy subjects. Furthermore, the data suggest that mucociliary transport is not the dominant clearance mechanism in small airways.

KEYWORDS: Cystic fibrosis, lung deposition, mucociliary clearance, small airways

iliary activity and rheology of airway surface liquid determine mucociliary clearance (MCC). MCC is one of the primary defence mechanisms in the lung to remove insoluble deposited material. This clearance mechanism is rapid, resulting in clearance of the larger airways within hours of inhalation and is considered to be completed within $24 \mathrm{~h}$ in healthy subjects. Any particles remaining in the lung after $24 \mathrm{~h}$ represent alveolar deposition [1, 2]. However, previous studies of healthy subjects using a shallow bolus technique as well as studies using extremely slow inhalation flow $\left(0.05 \mathrm{~L} \cdot \mathrm{s}^{-1}\right)$ and $6 \mu \mathrm{m}$ particles, resulting in particles deposited in the small airways, show that a considerable fraction of particles may be retained after $24 \mathrm{~h}$ [3-5] These studies provide evidence that the rapid clearance phase in the tracheobronchial region is followed by a second, slower clearance phase, though still more rapid than alveolar clearance. This intermediate clearance phase, proposed to represent clearance of particles deposited in the bronchiolar region, has been included in the revised dosimetric model for the human respiratory tract, adopted by the International Commission on Radiological Protection (ICRP) [6].
The inherited disease, cystic fibrosis $(\mathrm{CF})$, is characterised by production and accumulation of copious amounts of mucus with increased viscosity in the airways due to defective ion transport across the respiratory epithelium. This results in impaired MCC with recurrent bacterial infections, chronic inflammation, bronchiectasis and airway damage. This eventually culminates in respiratory failure and premature death. Early morphological changes in CF are first observed in the terminal bronchioles [7].

The most widely used method to study MCC in CF patients is the use of radiolabelled particles detected with the gamma camera technique. The rate of removal of the inhaled radiolabelled aerosol in the lung can be determined by serial gamma camera images for up to $24 \mathrm{~h}$. Such studies show that MCC is impaired in both central and peripheral regions [8, 9]. Impaired MCC is also characteristic in patients with primary ciliary dyskinesia, also known as immotile cilia syndrome, and in patients with chronic bronchitis. In these groups coughing clears centrally deposited particles quite effectively. The retained fraction at $24 \mathrm{~h}\left(\operatorname{Ret}_{24}\right)$ may even be smaller than in healthy subjects $[10,11]$.
AFFILIATIONS

*Division of Occupational Medicine, Dept of Public Health Science, Karolinska Institutet and Occupational and Environmental Health, Stockholm County Council,

\#Stockholm CF center, Dept of Paediatrics, Karolinska Institutet, Karolinska University Hospital, Huddinge site,

'Division of Inhalation Toxicology, Institute of Environmental Medicine, Karolinska Institutet, and ${ }^{+}$Swedish Radiation Protection Authority (SSI), Stockholm, Sweden.

CORRESPONDENCE

$M$. Lindström

Dept of Paediatrics B57 Karolinska University Hospital Huddinge Site

S-141 86 Stockholm

Sweden

Fax: 46858581410

E-mail: maria.lindstrom@phs.ki.se

Received

October 272003

Accepted after revision:

October 102004 
However, when particles are deposited in small airways, the retained fraction at 72 and $96 \mathrm{~h}$ is significantly larger in these patient groups, compared to that of healthy subjects [12, 13].

The current study investigated lung clearance in young adults with $\mathrm{CF}$, using a method depositing particles primarily in the small airways. The small airways were defined according to the Weibel model A [14], as generations 9-15, corresponding to the bronchioles, which have ciliated epithelium and are $<2 \mathrm{~mm}$ in diameter. The aims of the study were: 1) to test the hypothesis that the retained fraction of particles at $24 \mathrm{~h}\left(\operatorname{Ret}_{24}\right)$ is larger; and 2) that the clearance between day one and day 21 from small airways is slower in patients with CF compared to healthy subjects. It was also hypothesised that the $\operatorname{Ret}_{24}$ increases and the clearance between day 1 and 21 decreases with the severity of the lung disease.

\section{MATERIAL AND METHODS \\ Subjects}

Eleven patients (four males) attending the Stockholm CF center, aged $18.7 \pm 2.5$ (mean $\pm \mathrm{SD}$ ) yrs, with mild-to-moderate lung disease fulfilled the inclusion criteria and volunteered for the study. The inclusion criteria were: 1) diagnosis of CF in childhood with symptoms characteristic for $\mathrm{CF}$ and a positive sweat test $\left.\left(>80 \mathrm{Cl}^{-} \mathrm{mmoL} \cdot \mathrm{L}^{-1}\right)[15] ; 2\right)$ aged $\left.\geqslant 15 \mathrm{yrs} ; 3\right)$ a clinically stable condition; 4) no history of organ transplants; and 5) not pregnant. Anthropometric data, genotype, and Bhalla score [16], as well as current bacterial culture and inflammatory parameters (immunoglobulin G) together with the lung function data taken at the onset of the study are shown in table 1 . All patients were tested for 32 common CF mutations, including the three most prevalent in Sweden, $\Delta F 508$, 3659delC and 394delTT. Three patients were considered chronically colonised solely with Staphylococcus aureus and seven patients with Pseudomonas aeruginosa, of whom two were also colonised with $S$. aureus, in their lower airways. A patient was considered chronically colonised when the same pathogen was regularly isolated from sputum or nasopharyngeal swabs for a period $>6$ consecutive months. All patients were on oral mucolytic agents (bromhexin and acetylcystein) and inhaled salbutamol and acetylcystein once to twice daily combined with mucus mobilising and evacuating exercise as part of their daily physiotherapy. None of the patients were prescribed systemic corticosteroids or received nebulised rhDNase or nebulised antibiotics. Despite being in a clinically stable condition, three of the CF patients (No. 6, 9 and 11) showed slightly elevated C-reactive protein, No. 6 being on an i.v. antibiotic treatment initiated at slight signs of low-grade infection [17]. Two patients with moderate lung disease coughed spontaneously during the exposure and reported spontaneous cough during the study period of 21 days. The other CF patients only coughed voluntarily together with their daily physiotherapy. Their coughs were not monitored in detail over time.

The pulmonary function was evaluated by forced expirograms (Lung Function Laboratory 2100; SensorMedics, Anaheim, CA, USA). The same technical assistant coached each CF patient. The airway resistance (Raw), was measured using a panting technique within a whole-body plethysmograph (Transmural Body Box 2800; SensorMedics). All lung function parameters were determined according to the criteria proposed by QUANJER et al. [18]. Prior to the clearance, data were evaluated and the patients were categorised on the basis of their lung function, one group with mild lung disease (forced expiratory volume in one second (FEV1) $>70 \%$ of predicted; $n=6$ ), and one group with moderate-to-severe lung disease (FEV1 $<70 \%$ pred; $\mathrm{n}=5$ ).

The study was approved by the Ethics Committee of Human Research at the Karolinska University Hospital and the Isotope Committee at the Karolinska Hospital. All patients and healthy subjects were provided with written information about the study and informed that the exposure of radioactivity to the lung was lower than the radiation dose of a conventional chest radiograph. All the patients and healthy subjects gave their written informed consent to participate in the study. Parental consent was obtained for the patients aged $<18$ yrs.

\section{Study design}

Sample size calculation based on variability data from the pooled SD in a previous study comparing clearance between healthy subjects and patients with chronic bronchitis [19], showed a demand of 12 individuals in each group to detect a difference of one SD with a power of $80 \%$. The authors were finally able to recruit $11 \mathrm{CF}$ patients. The CF patients and the healthy subjects inhaled $6-\mu \mathrm{m}$ monodisperse Teflon particles labelled with ${ }^{111}$ Indium with an extremely slow inhalation flow, $0.05 \mathrm{~L} \cdot \mathrm{s}^{-1}$, giving a deposition mainly in the small airways. Radioactivity over the mouth, throat, lung and stomach was measured immediately after the inhalation of the test particles. Lung retention was measured at $24 \mathrm{~h}$, and at day 7, 14 and 21 post-inhalation. Correction was made for background activity and physical decay of the radionuclide. The regional deposition data were estimated using a model developed at the Karolinska Institutet. In the evaluation of the data, the studied period was divided into two phases. A first rapid clearance phase was defined as the clearance between 0 and $24 \mathrm{~h}$, representing mostly the large- and medium-sized airways and, a second slow clearance phase, defined as the clearance between day 1 and day 21, representing mostly the small airways.

For ethical reasons, present data was compared with previous data from healthy subjects of similar age from the current authors' previous studies. They were nonsmokers, nonasthmatics who had normal lung function. Both the healthy subjects and the CF patients were exposed using the same equipment for particles production procedure and inhalation. Identical inhalation pattern was used and radioactivity was measured in the lung with the same detectors throughout the study in both groups [5,20,21]. The average anthropomorphic and lung function data (mean $\pm \mathrm{SD}$ ) of the healthy subjects are given in table 1 .

\section{Inhalation of the test particles and measurement of radioactivity}

The Teflon particles were produced and labelled with ${ }^{111} \mathrm{In}$ (half-life $68 \mathrm{~h}$ ) by a spinning disc technique [22]. The mean aerodynamic diameter was calculated to be $6.2 \mu \mathrm{m}$ [20]. The subjects wore a nose-clip and inhaled the particles in a sitting position. The subjects first made a moderately deep exhalation outside the chamber and then inhaled as deep as they could from the chamber. The flows were monitored using a pneumotachograph, placed between the aerosol chamber and 
TABLE 1 Subject characteristics and lung function data

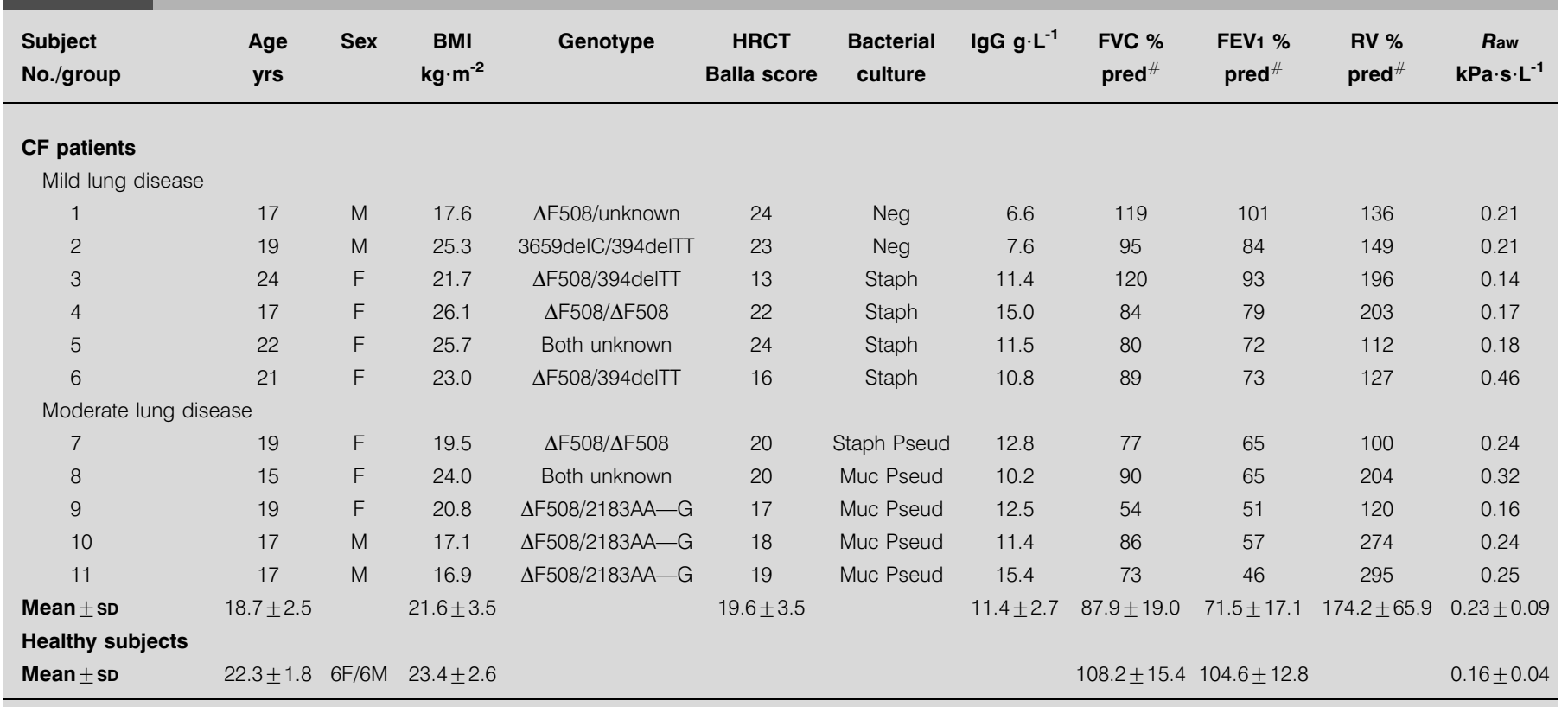

BMI: body mass index (weight/height² ${ }^{2}$; HRCT: high-resolution computer tomography of the lungs, the findings scored according to BHALLA [16]; IgG: immunoglobulin G; FVC: forced vital capacity; pred: predicted; FEV1: forced expiratory volume in one second; RV: residual volume; Raw: airway resistance; CF: cystic fibrosis; M: male; F: female; Neg: negative, normal flora; Staph: Staphylococcus aureus; Muc Pseud: Mucoid Pseudomonas aeruginosa; Pseud: Nonmucoid P.aeruginosa. \#: predicted values according to QUANJER et al. [18]. CF patients: $n=11$; healthy subjects: $n=12$.

the mouthpiece, and were recorded graphically. Between each particle inhalation from the chamber, the subject inhaled and exhaled several times outside the chamber. The mean inhalation flow was $0.045 \pm 0.003 \mathrm{~L} \cdot \mathrm{s}^{-1}$. Exhaled radioactivity has earlier been shown to be $0-2 \%$ [23]. The duration of the exposure was $6.3 \pm 2 \mathrm{~min}$ and the duration of each inhalation was approximately $30 \mathrm{~s}$.

Immediately following the inhalation and $24 \mathrm{~h}$ later the radioactivity was measured using two $127 \times 51 \mathrm{~mm} \mathrm{NaI}$ crystals fitted with collimators. The average radioactivity deposited in the lung was $0.1 \mathrm{MBq}$. The authors believe that hot spot deposition was probably limited as the particles settle mainly by sedimentation and not by impaction. The radioactivity was also measured at $24 \mathrm{~h}$ and at day 7 after inhalation using the whole-body scanner with three large NaI detectors, at the Swedish Radiation Protection Institute [20, 24]. At day 7, 14 and 21, when the radioactivity in the gastrointestinal tract was insignificant compared to the lung radioactivity, a more sensitive lung counter was used with the subject in supine position.

\section{Theoretical calculation of lung deposition}

The theoretical deposition data were examined using a model developed at the Karolinska Institutet, the KI-model, which is based on the equations for impaction and sedimentation from the model of the Task Group on Lung Dynamics [25], and on the human airway model A (comprising generations 0-23 of which generations 0-16 are ciliated), proposed by WEIBEL [14]. In the KI-model, inhalation parameters such as airway dimensions, tidal volume, inspiratory flow, post-inspiratory pause, and expiratory time can be changed over a wide range.
The diameters of generations $0-23$ were changed by the same factor and deposition was calculated for each generation. The factor was chosen so that it gave a calculated airway resistance (using Poiseuille's law), which was the same as the one measured in the test subjects minus $0.05 \mathrm{kPa} \cdot \mathrm{s} \cdot \mathrm{L}^{-1}$, representing the airway resistance in mouth, throat, and larynx. The KI-model has shown to be highly reproducible and there are no major differences when comparing prediction for the KI-model with other more elaborate models, such as the human respiratory tract model modified in the ICRP publication 66 and the University of Salzburg model (USBG) using the Monte Carlo particle transport and deposition code (IDEAL) [24]. The two CF groups and the healthy subjects had an average Raw of $0.228,0.242$ and $0.155 \mathrm{kPa} \cdot \mathrm{s} \cdot \mathrm{L}^{-1}$, respectively. The dimension factors $0.56,0.55$ and 0.65 , respectively, were used to mimic airway resistance, an inhalation flow of $0.045 \mathrm{~L} \cdot \mathrm{s}^{-1}$, a pause of $2 \mathrm{~s}$, and a tidal volume of $1.25,1.04$ and $1.50 \mathrm{~L}$, respectively.

\section{Statistical analyses}

The descriptive data are expressed as mean \pm SD and the results as mean $(95 \%$ confidence interval (CI)) when appropriate. Nonparametric rank sum test for evaluation of differences between the groups and the Spearman correlation coefficient were used; $\mathrm{p}<0.05$ was considered significant. Measurements were adjusted at 2 and 3 weeks using interpolation to day 14 and 21 , in the few cases when the measured point differed by 1 day.

\section{RESULTS}

Mean FEV1 in the CF patients was $2.4 \mathrm{~L}$ (1.7-3.4) corresponding to $72 \pm 17 \%$ pred, compared to $4.2 \mathrm{~L}$ (3.2-5.9) 
corresponding to $105 \pm 13 \%$ pred in the healthy subjects. The CF patients with moderately severe lung disease showed growth of $P$. aeruginosa in their sputum sample, taken at the start of the study, compared with none in the CF group with mild lung disease (table 1), although there were patients in the latter group who were considered chronic colonised with $P$. aeruginosa, in their lower airways.

The calculated regional deposition in different airway regions according to the theoretical model showed that the main fraction was deposited in the small conducting airways (generations 9-15). The deposition pattern did not differ between the healthy subjects and the CF patients, nor did it differ between the two CF groups according to their lung disease (fig. 1). $\operatorname{Ret}_{24}$ was significantly higher $(\mathrm{p}<0.001)$ in the CF patients, 67\% (95\% CI 58-76), compared to healthy subjects, $48 \%$ (95\% CI 42-53). The difference in lung retention over time persisted and was still higher at the last measured point at day $21,34 \%$ (95\% CI 30-38) and 26\% (95\% CI 21-31) $(\mathrm{p}<0.01)$, respectively (fig. 2). The cleared fraction between day 1 and day 7 was greater among the CF patients, 22\% (95\% CI 15-29) compared to that of the healthy subjects, $14 \%$ (95\% CI 12-16) $(\mathrm{p}<0.05)$.

There was a significant clearance after $24 \mathrm{~h}$ in all the subjects. The healthy subjects and the CF patients showed a cleared fraction during 21 days of $55 \%$ and $50 \%$ of Ret $_{24}$, respectively. There was no statistical difference detected by linear regression of the slope between day 7 and 21. Lung retention at day 7, 14 and 21 , in \% of $\operatorname{Ret}_{24}$, were similar between the healthy subjects and the two CF groups (fig. 3). There was a slight difference, but a larger variation, in $\operatorname{Ret}_{24}$ between the two CF groups, $63.8 \pm 16.4 \%$ (mean $\pm \mathrm{SD}$ ) in patients with mild lung disease, compared to $70.8 \pm 5.9 \%$ in patients with moderately severe lung disease, $(p=0.4)$. After $24 \mathrm{~h}$ the retention at day 7,14 and 21 differed between the two CF groups $(p<0.01)$. Patient No. 2 in the CF group with mild lung disease had a $\operatorname{Ret}_{24}$ of $88 \%$, but

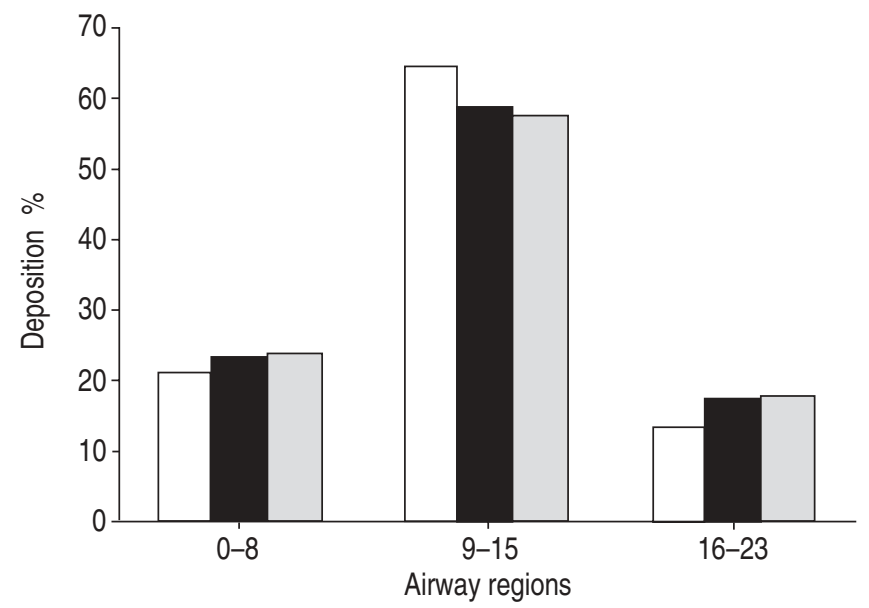

FIGURE 1. Calculated regional deposition, in large airways (representing generations 0-8), small airways (representing generations 9-15) and alveolar region (representing generations 16-23) in percentage of total lung deposition of $6-\mu \mathrm{m}$ particles inhaled with extremely slow inhalation flow $\left(0.05 \mathrm{~L} \cdot \mathrm{s}^{-1}\right)$ at mean airway resistance in the healthy subjects $(\square)$ and the two cystic fibrosis (CF) groups (

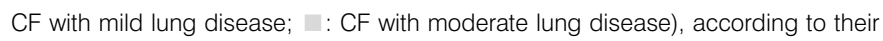
lung function.

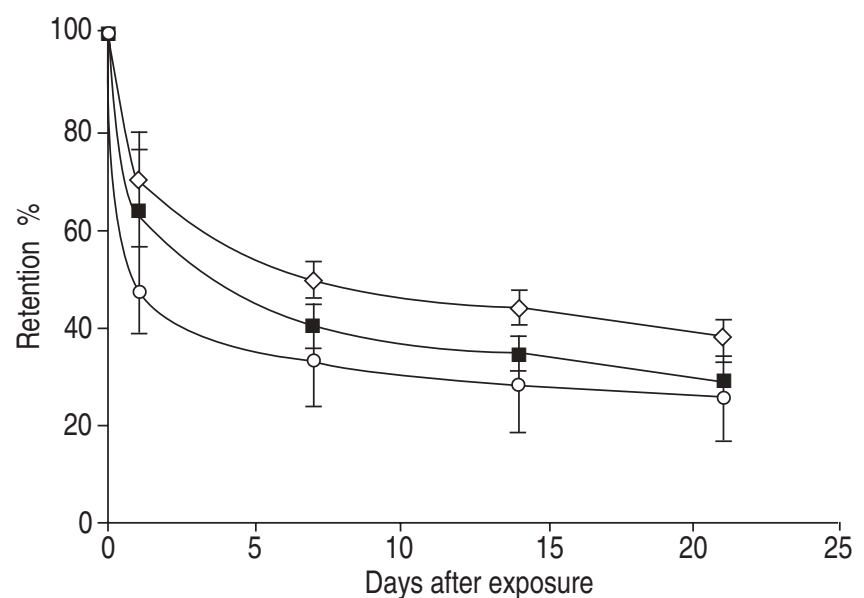

FIGURE 2. Clearance curves using extremely slow inhalation flow $\left(0.05 \mathrm{~L} \cdot \mathrm{s}^{-1}\right)$ comparing cystic fibrosis patients with mild ( $>70 \%$ forced expiratory volume in one second (FEV 1$)$ pred; $\mathbf{\square})$ and moderate $(<70 \%$ FEV 1 pred; $\diamond)$ lung disease to the healthy subjects $(\bigcirc)$.

after 1 week the retained fraction had reached the same level as that of the other patients in the same group. Another patient (No. 6), on i.v. antibiotics (the treatment was ended after 7 study days), as well as the patient (No. 9) who had biochemical signs of exacerbation, did not differ from the others in clearance activity, e.g. were not in the outer range.

\section{DISCUSSION}

The objective of this study was to investigate long-term clearance from small airways in patients with CF. The most important and unexpected finding was similar clearance in the CF patients and the healthy subjects after day 7 up to the last measured point at day 21. Furthermore, an increased fraction of retained particles was found at $24 \mathrm{~h}$ in the $\mathrm{CF}$ patients compared to that in the healthy subjects.

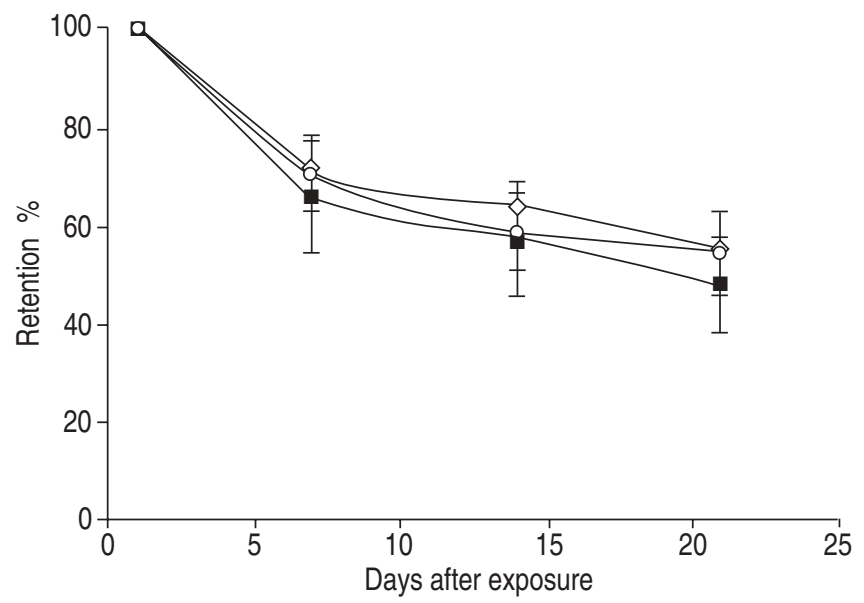

FIGURE 3. The slow clearance phase using extremely slow inhalation flow $\left(0.05 \mathrm{~L} \cdot \mathrm{S}^{-1}\right)$. Clearance as a function of time normalised to retention at $24 \mathrm{~h}$, comparing the cystic fibrosis patients with mild $(>70 \%$ forced expiratory volume in one second (FEV1) pred; $\mathbf{\square})$ and moderate ( $<70 \%$ FEV 1 pred; $\diamond)$ lung disease to the healthy subjects $(\bigcirc)$. Standardisation to $24 \mathrm{~h}$ is a standard procedure to omit the first rapid clearance phase. 
The estimated lung deposition of the inhaled particles was to a large extent deposited in the small airways, and the calculated regional deposition predicted very small differences between the CF patients (irrespective of lung disease) and the healthy subjects. The large $\operatorname{Ret}_{24}(67 \%$ in the CF patients compared to $48 \%$ in the healthy subjects) also indicated a larger deposition in the small airways than that found using normal inhalation flow. If the fraction of particles were deposited more centrally in the lungs, the $\operatorname{Ret}_{24}$ would probably be smaller in the CF patients than in healthy subjects [26], since the CF patients probably clear the central airways faster by their daily physiotherapy and voluntary coughing. If the fraction of particles were deposited in the alveolar region, then the clearance after $24 \mathrm{~h}$ would have been much slower than that measured in the current study [27]. In patients with asthma, chronic bronchitis or $\mathrm{CF}$, the airways may be narrowed by bronchospasm, oedema or mucus secretion, resulting in a turbulent flow that increases impaction in the larger airways and thereby reduces the penetration to the small airways of the lung. In contrast, in the small airways the airflow is slow due to the large total cross-sectional area and particle sediment by gravity onto the airway surface. By using extremely slow inhalation flow (ESI) and rather large particles, the fraction deposited in small airways increases by sedimentation. Even if there was a difference in FEV1\% pred and airway resistance between the CF patients and the healthy subjects the ESI has shown to be robust and rather insensitive to airway obstruction and uneven ventilation [28].

The larger $\operatorname{Ret}_{24}$ in the CF patients compared to the healthy subjects was expected. There was also a larger variation in Ret $_{24}$ in the CF patients compared to later time points and to the healthy subjects at all time points. Furthermore, there was a larger difference between $\operatorname{Ret}_{24}$ and retention of particles at day 7 in the CF patients. The authors' interpretation was that their rapid clearance phase not was completed within $24 \mathrm{~h}$, but continued and was probably completed sometime before day 7. A larger retained fraction at $24 \mathrm{~h}$ in the CF patients could also contribute to the faster elimination during day 1-7. In healthy subjects the rapid clearance phase (from deposition to the first $24 \mathrm{~h}$ ) is governed mainly by the mucociliary escalator with normal mucus and cilia activity. In CF patients this clearance mechanism is disturbed, and the CF patients might compensate their impaired MCC by a more unpredictable cough clearance. Cough probably has greater impact on clearance from the larger airways where the necessary airflow velocity is achieved, allowing the effects on the rapid clearance phase. The patient (No. 3), who had a $\operatorname{Ret}_{24}$ of $88 \%$, and then cleared $50 \%$ of the retained fraction to the next measured point at day 7 , might be an illustration of this cough clearance as a compensatory mechanism for impaired MCC.

The slow clearance phase, measured from day 7, in percentage of $\operatorname{Ret}_{24}$, was similar in CF patients and healthy subjects. All subjects continued to clear their airways with the same velocity and, on average $50 \%$ of the retained particles at $24 \mathrm{~h}$ cleared during this period. The similarity between the CF patients and the healthy subjects may be attributable to insufficient power. To increase the power of the differences between the $\mathrm{CF}$ patients and the healthy subjects they were also tested with an independent $t$-test with the same result. MCC is probably not the primary rate determining factor for clearance from the small airways. The contribution of cough to clearance in the small airways is probably limited, though cough as a mechanical force may influence other secondary clearance mechanisms. In a study using a similar method to the current study, bronchiolar clearance was studied for up to $72 \mathrm{~h}$ in children with Duchenne muscular dystrophy, who had healthy lungs but severely reduced muscular strength. There was a significantly lower retention at $72 \mathrm{~h}$ (but not after 24 or $48 \mathrm{~h}$ ) than in the control experiment with continuous positive airway pressure treatment, suggesting that a mechanical force can influence the long-term clearance from small airways [29]. Other clearance mechanisms such as phagocytosis by alveolar macrophages, penetration of the test particles though the epithelium or re-transfer with a time delay of particles onto the gel layer, may also contribute to clearance during the slow clearance phase [30].

Several factors may be associated with lung clearance such as age, sex, BMI, lung function and airway resistance. The age and sex between the CF and the healthy subjects where similar, although they where not individually matched. The BMI differed, but this factor was included as a marker for nutritional status as a consequence of the CF disease and not as a factor correlated to the lung retention. However, it is still possible that BMI itself, separate from the causality of the $\mathrm{CF}$ disease, has an independent effect that may have influenced the observations. The present authors have however, not found any correlation between BMI and lung retention in healthy subjects.

FEV1 \% pred and airway resistance also differed between CF patients and healthy subjects. As discussed earlier these factors have limited effect on the deposition. However, FEV1 \% pred as an individual factor, predicting the lung clearance, was uncertain, and the square correlation coefficient $\left(r^{2}\right)$ between FEV1 \% pred and $\operatorname{Ret}_{24}$ was 0.27 . The CF patients were divided into two subgroups according to their lung function, measured as $\mathrm{FEV} 1 \%$ pred to test if a more serious lung disease was associated with a higher lung retention on group level. The current bacterial airway colonisation showed an association with decreased FEV1 \% pred. The CF group with moderately severe lung disease (FEV $1<70 \%$ pred) had a higher burden of $P$. aeruginosa in their airways, which is in concordance with previous findings [31]. There was a nonsignificant difference in Ret $_{24}$ between the two CF groups. This was probably due to the large variability in the rapid clearance phase (reflecting clearance from large airways). At later measured time points this difference became significant, with larger lung retention in $\%$ of deposition in CF patients with moderately severe lung disease. It seems that patients with a slower clearance during the first $24 \mathrm{~h}$, can catch up due to a faster clearance during the first week, but that the degree of the lung disease influences the lung retention during the rapid clearance phase (the first week). The patients with the more severe lung disease have a longer exposure to inhaled materials. Whether the prolonged rapid clearance phase is caused by the bacterial colonisation in the airways, or if the slower clearance is primarily associated with the disease, $\mathrm{CF}$, has to be confirmed. The bronchodilatator terbutaline, that has a known stimulating effect on short-term MCC, may have influenced the result of the rapid clearance phase but should not affect the slow clearance phase from small airways [32]. 
Earlier clearance studies, mostly gamma camera studies, on CF patients, have used normal inhalation flow. When normal inhalation flow $\left(>0.2 \mathrm{~L} \cdot \mathrm{s}^{-1}\right)$ is used, the particles are deposited mainly in larger airways or in the alveolar region and, only to a small extent in the small airways, independent of particle size [24]. The breathing pattern and the particle size used highly influence the site of the deposition within the lungs. The deposition is probably the most important determinant of measured MCC $[26,33]$. It is therefore difficult to evaluate different studies of MCC in which the site of the deposition might have varied. To the best of the authors' knowledge this is the first study on long-term clearance in patients with CF.

\section{Conclusions}

The retention of particles in \% of deposition at $24 \mathrm{~h}$ was larger in the CF patients compared to the healthy subjects. The CF patients showed faster clearance than the healthy subjects between day 1 and 7, indicating that the rapid clearance phase continued after $24 \mathrm{~h}$ compensated by cough to complete clearance of the large to medium-sized airways. Both the CF patients and the healthy subjects showed a significant clearance during day 7 and 21, representing clearance mainly from the small airways. This slow clearance phase did not differ between the CF patients and the healthy subjects. The CF patients may have a changed mucus rheology that can affect the mucociliary transport but this mechanism seems not to be the crucial mechanism for clearance from the small airways. The severity of the lung disease seemed to influence the rapid clearance phase but not the slow clearance phase, with a tendency of a larger retained fraction after $24 \mathrm{~h}$ associated with a lower FEV1 \% pred.

With extremely slow inhalation flow it is possible for $6-\mu \mathrm{m}$ particles to target small airways. This inhalation methodology may be of clinical utility in selectively targeting small airways for the deposition of therapeutic drugs such as antibiotics or gene therapy. Further studies are needed to confirm the hypothesis that mechanisms other than mucociliary clearance dominate in the small airways. A study similar to the present investigating long-term clearance from small airways in patients with impaired ciliary activity may be helpful in elucidating clearance mechanisms.

\section{ACKNOWLEDGEMENTS}

This study was supported by grants from the Swedish Heart and Lung foundation, the European Commission (contract no. FIS5-1999-00214, BIODOSE), Karolinska Institutet foundation and, from Free Mason in Stockholm Foundation for Children's Welfare. The authors would like to thank L-L. Söderström, Dept of Clin. Physiology, South Stockholm General Hospital, for technical assistance with the lung function test, I. Östergren, Swedish Radiation Protection Authority (SSI) for skilful technical assistance, U. Bergsten for secretary assistance and M. Lundberg for guidance of radioactivity measurements, Institute of Environmental Medicine, Karolinska Institutet.

\section{REFERENCES}

1 Lippman M, Albert RE. The effect of particle size on the regional deposition of inhaled aerosols in the human respiratory tract. Am Ind Hyg Assoc J 1969; 30: 257-275.
2 Camner P, Philipson K. Human alveolar deposition of 4 micron teflon particles. Arch Environ Health 1978; 33: 181-185.

3 Stahlhofen W, Scheuch G, Bailey MR. Measurement of the tracheobronchial clearance of particles after aerosol bolus inhalation. Ann Occup Hyg 1994; 38, Suppl: 1, 189-196.

4 Scheuch G, Philipson K, Falk R, et al. Retention of particles inhaled in boli with and without induced bronchoconstriction. Exp Lung Res 1995; 21: 901-916.

5 Svartengren M, Sommerer K, Scheuch G, et al. Comparison of clearance of particles inhaled with bolus and extremely slow inhalation techniques. Exp Lung Res 2001; 27: 367-386.

6 Task Group of the International Commission on Radiological Protection (ICRP). Human Respiratory Tract Model for Radiological Protection Annals ICRP Publication 66. 1994; 24, 1-3, 1-482.

7 Khan TZ, Wagener JS, Bost T, Martinez J, Accurso FJ, Riches DW. Early pulmonary inflammation in infants with cystic fibrosis. Am J Respir Crit Care Med 1995; 151: 1075-1082.

8 Robinson M, Eberl S, Tomlinson C, et al. Regional mucociliary clearance in patients with cystic fibrosis. J Aerosol Med 2000; 13: 73-86.

9 Regnis JA, Zeman KL, Noone PG, Knowles MR, Bennett WD. Prolonged airway retention of insoluble particles in cystic fibrosis versus primary ciliary dyskinesia. Exp Lung Res 2000; 26: 149-162.

10 Ruusa J, Svartengren M, Philipson K, Camner P. Tracheobronchial particle deposition and clearance in immotile cilia syndrome patients. J Aerosol Med 1993; 6: 89-98.

11 Ericsson $\mathrm{CH}$, Svartengren $\mathrm{K}$, Svartengren $\mathrm{M}$, et al. Repeatability of airway deposition and tracheobronchial clearance rate over three days in chronic bronchitis. Eur Respir J 1995; 8: 1886-1893.

12 Svartengren K, Philipson K, Svartengren M, Nerbrink O, Camner P. Clearance in smaller airways of inhaled 6micron particles in subjects with immotile-cilia syndrome. Exp Lung Res 1995; 21: 667-682.

13 Svartengren K, Philipson K, Svartengren M, Anderson M, Camner P. Tracheobronchial deposition and clearance in small airways in asthmatic subjects. Eur Respir J 1996; 9: 1123-1129.

14 Weibel ER. Morphometry of the Human Lung. New York, Springer-Verlag, Berlin Academic Press, 1963; pp. 136143.

15 Gibson LE, Cooke RE. A test for concentration of electrolytes in sweat in cystic fibrosis of the pancreas utilizing pilocarpine by iontophoresis. Paediatrics 1959; 23: 545-549.

16 Bhalla M, Turcios N, Aponte V, et al. Cystic fibrosis: scoring system with thin-section CT. Radiology 1991; 179: 783-788.

17 Strandvik B, Hjelte L, Malmborg A-S. The Swedish experience. In: Cystic Fibrosis Pulmonary Infections: Lessons from Around the World. Bauernfeind A, Marks MI, Strandvik B, eds. Basel, Birkhäuser, 1996; pp. 293-302.

18 Quanjer PH, Tammeling GJ, Cotes JE, Pedersen OF, Peslin R, Yernault JC. Lung volumes and forced ventilatory flows. Report Working Party Standardization of Lung Function Tests, European Community for Steel and Coal. 
Official Statement of the European Respiratory Society. Eur Respir J 1993; 6: Suppl. 16, 5-40.

19 Svartengren K, Ericsson CH, Svartengren M, Mossberg B, Philipson K, Camner P. Deposition and clearance in large and small airways in chronic bronchitis. Exp Lung Res 1996; 22: 555-576.

20 Falk R, Philipson K, Svartengren M, Jarvis N, Bailey M, Camner P. Clearance of particles from small ciliated airways. Exp Lung Res 1997; 23: 495-515.

21 Philipson K, Falk R, Svartengren M, et al. Does lung retention of inhaled particles depend on their geometric diameter? Exp Lung Res 2000; 26: 437-455.

22 Camner P. The production and use of test aerosols for studies of human tracheobronchial clearance. Environ Physiol Biochem 1971; 1: 37-154.

23 Anderson M, Philipson K, Svartengren M, Camner P. Human deposition and clearance of 6-micron particles inhaled with an extremely low flow rate. Exp Lung Res 1995; 21: 187-195.

24 Falk R, Philipson K, Svartengren M, et al. Assessment of long-term bronchiolar clearance of particles from measurements of lung retention and theoretical estimates of regional deposition. Exp Lung Res 1999; 25: 495-516.

25 Task Group on Lung Dynamics. Deposition and retention models for internal dosimetry of human respiratory tract. Health Phys 1966; 12: 173-207.

26 Robinson M, Bye PT. Mucociliary clearance in cystic fibrosis. Pediatr Pulmonol 2002; 33: 293-306.
27 Philipson K, Falk R, Gustafsson J, Camner P. Long-term lung clearance of 195Au-labeled teflon particles in humans. Exp Lung Res 1996; 22: 65-83.

28 Svartengren K, Svartengren M, Philipson K, Barck C, Bylin G, Camner P. Clearance in small ciliated airways in allergic asthmatics after bronchial allergen provocation. Respiration 1999; 66: 112-118.

29 Klefbeck B, Svartengren K, Camner P, et al. Lung clearance in children with Duchenne muscular dystrophy or spinal muscular atrophy with and without CPAP (continuous positive airway pressure). Exp Lung Res 2001; 27: 469484.

30 Sturm R, Hofmann W. Mechanistic interpretation of the slow bronchial clearance phase. Radiat Prot Dosimetry 2003; 105: 101-104.

31 Baltimore RS, Christie CD, Smith GJ. Immunohistopathologic localization of Pseudomonas aeruginosa in lungs from patients with cystic fibrosis. Implications for the pathogenesis of progressive lung deterioration. Am Rev Respir Dis 1989; 140: 1650-1661.

32 Svartengren K, Philipson K, Svartengren M, Camner P. Effect of adrenergic stimulation on clearance from small ciliated airways in healthy subjects. Exp Lung Res 1998; 24: 149-158.

33 Ilowite JS, Smaldone GC, Perry RJ, Bennett WD, Foster WM. Relationship between tracheobronchial particle clearance rates and sites of initial deposition in man. Arch Environ Health 1989; 44: 267-273. 